
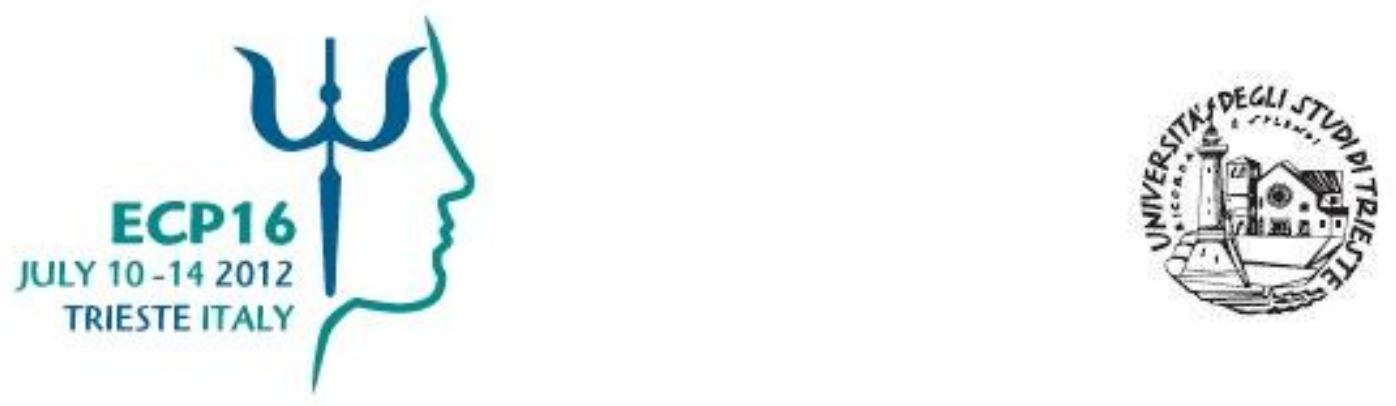

\title{
16th European Conference on Personality
}

\author{
10-14 July 2012
}

Trieste, Italy

Dear Colleagues,

on behalf of organizing and scientific committees, we are honored and pleased to announce you that the 16th European Conference on Personality (ECP16) will be held in Trieste (Italy), from Tuesday 10th to Saturday 14th July, 2012.

The conference, organized by the European Association of Personality Psychology (EAPP) and the Department of Psychology "G. Kanizsa", University of Trieste, will host colleagues from different countries all around the world. As you might already know, EAPP conferences promote contemporary research findings and exchange of ideas among different fields related to personality psychology. In line with these aims, ECPI6 scientific program will include contributions on distinctive personality issues as personality development, personality and social psychology, personality and work, methodological issues in personality assessment, personality and culture, personality and politics, as well as behavioral genetics, personality and neuroscience, personality and cognitive processes, and more.

ECPI6 will host six leading scholars as keynote speakers and organize symposia and papers in a series of five parallel sessions; three sessions will be fully dedicated to poster presentations. Lastly, during ECP16, a life time achievement award and, for the first time, an early career award will be conferred to two distinguished European personality psychologists.

EAPP conferences are among the most important venues to present and hear about current personality research. Furthermore, Trieste offers a pleasant and relaxed atmosphere to meet leading experts as well as emerging and young students in personality psychology.

We look forward to meeting you in Trieste!

Lisa Di Blas and Andrea Carnaghi

\section{Visit the website for more information http://www.theoffice.it/ecp16}

